\title{
Influence of operative timing on the early post-operative radiological and clinical outcome after kyphoplasty
}

\author{
Yannick Palmowski ${ }^{1}$ (I) Sophie Balmer ${ }^{1}$. Justus Bürger ${ }^{1}$. Friederike Schömig ${ }^{1} \cdot$ Zhouyang Hu$^{1}$. \\ Matthias Pumberger ${ }^{1}$
}

Received: 24 March 2020 / Revised: 10 May 2020 / Accepted: 31 May 2020 / Published online: 15 June 2020

(c) The Author(s) 2020

\begin{abstract}
Purpose To clarify the relationship between operative timing and the early post-operative radiological and clinical outcome after kyphoplasty.

Methods We conducted a retrospective cohort study including patients who underwent kyphoplasty of a single vertebra. Patients were divided into three groups (acute [ $<2$ weeks], subacute [2-6 weeks] or chronic [6-51 weeks]) based on the interval between fracture and surgery. The relative vertebral body height (VBH) and local kyphotic angle (LKA) of the fractured vertebra (measured on plain radiographs) as well as pain and use of analgesics were compared pre- and post-operatively (day 2) and between the groups.

Results A total of 230 patients (100 with acute, 91 with subacute and 39 with chronic fractures) with fractures from T4 to L5 were included. In all groups, there was a significant post-operative improvement in the anterior (8.9-12.9\%) and middle (10.7-13.4\%) VBH (all groups: $p<0.001$ ), LKA (acute: $3.8^{\circ}, p<0.001$; subacute: $4.3^{\circ}, p<0.001$; chronic: $1.7^{\circ}, p=0.046$ ) and pain. The use of analgesics significantly decreased post-operatively in the acute and subacute groups, but did not significantly change in the chronic group. Patients from acute $(p=0.042)$ and subacute $(p=0.027)$ groups showed significantly better post-operative correction of the LKA than the chronic group.

Conclusion Kyphoplasty is effective for vertebral height restoration as well as pain relief for both acute, subacute and chronic fractures. However, the achievable correction of the fracture-related local kyphosis decreases significantly after 6 weeks. Therefore, we recommend making a final decision about conservative vs. operative treatment within 6 weeks to ensure better height restoration in surgically treated patients.
\end{abstract}

Keywords Kyphoplasty $\cdot$ Vertebral compression fracture $\cdot$ Osteoporosis $\cdot$ Operative timing

Yannick Palmowski

yannick.palmowski@charite.de

Sophie Balmer

sophie.balmer@charite.de

Justus Bürger

justus.buerger@charite.de

Friederike Schömig

friederike.schoemig@charite.de

Zhouyang $\mathrm{Hu}$

zhouyang.hu@charite.de

Matthias Pumberger

matthias.pumberger@charite.de

1 Charité - Universitätsmedizin Berlin, corporate member of Freie Universität Berlin, Humboldt-Universität zu Berlin, and Berlin Institute of Health, Center for Musculoskeletal Surgery, Chariteplatz 1, 10117 Berlin, Germany

\section{Introduction}

Osteoporotic fractures are a major cause of morbidity in elderly patients [1-3]. There are currently over 8.9 million osteoporotic fractures per year worldwide, and this number is likely to increase in near future because of the aging the population $[4,5]$. This makes their optimal treatment a highly relevant medical challenge [6].

Most of these fractures are vertebral compression fractures (VCF) [7]. For various reasons, these fractures can lead to a high burden for the affected patients. Studies have shown that a relevant percentage of patients with VCFs continue to have pronounced pain and reduced quality of life even after 1 year [8]. In addition, vertebral fractures can result in severe fracture-related deformities, which can be both aesthetically disturbing and functionally limiting for 
affected patients. Thus, an optimal treatment should ideally offer both efficient pain relief and a local restoration of the fracture-related deformity.

Both conservative and operative treatments are available to manage patients with VCF. In many cases, intensive conservative treatment protocols allow good pain relief in the long term [9]. For surgical alternatives, the standard procedure for treatment of stable VCF is percutaneous balloon kyphoplasty. In addition to quick pain reduction, this approach also allows local height restoration of the fractured vertebra by inflating it with a balloon and subsequently stabilizing it with cement. Current guidelines mainly recommend an initial conservative treatment attempt and to consider surgical options only in cases of persistent pain [10]. However, the optimal duration of such conservative treatment remains unclear. It seems plausible that a long interval between a fracture occurrence and surgery might limit the achievable height restoration because of fracture consolidation. Therefore, it might be favourable for an optimal long-term outcome not to extend conservative treatment too long. However, there are no robust data on the interval during which kyphoplasty should ideally be performed. The available studies are rather small (each $<100$ patients) and they do not allow reliable recommendations to be made on the optimal operative timing. For instance, two studies report a better height restoration in fresh fractures (treated within 4 weeks), but did not examine possible differences between subacute and chronic fractures [11, 12]. Consequently, national and international guidelines do not provide consistent recommendations, with some guidelines recommending a conservative treatment attempt of 2-3 weeks while others do not provide any recommendations regarding the duration at all $[10,13,14]$.

In the present study, we aimed to investigate the relationship between the time of surgical intervention and radiological as well as clinical outcomes after kyphoplasty by retrospectively analysing data from single-level kyphoplasties of the thoracolumbar spine.

\section{Materials and methods}

\section{Patients}

We included patients who underwent kyphoplasty of a single lumbar or thoracic VCF in our orthopaedic department between January 2012 and December 2018. Patients with a malignant neoplasia in the respective vertebra or patients who had multiple fractures that were treated at the same time were excluded from the study. The included patients were divided into three groups depending on the interval between injury and operation: acute group $(<2$ weeks), subacute group (2-6 weeks) and chronic group ( $>6$ weeks).
The time of injury was defined as the time of symptom onset reported by the patient, and only patients who had reported a clear time of symptom onset were included in the study. Following German guidelines for the treatment of osteoporotic vertebral compression fractures, all patients received an initial conservative treatment attempt [10]. If this conservative attempt failed and no sufficient pain reduction was reached, surgical treatment was recommended.

\section{Surgical procedure}

All patients were treated in a prone position under general anaesthesia using a bipedicular approach and biplanar C-arm imaging guidance. After insertion of the balloons, the inflation was controlled visually and manometrically. In all cases, the inflation was stopped at a pressure of 300 PSI, when the balloon made contact with either of the endplates or at total restoration of the vertebral body height. After allowing the bone cement to reach a toothpaste-like viscosity, the cavity was filled with polymethylmethacrylate cement. The patients stayed in a prone position for at least $15 \mathrm{~min}$ after cement administration so that the curing process would not be compromised. All patients received perioperative intravenous antibiotics.

\section{Radiographic parameters}

Two researchers especially trained in the measurement of radiological spinal parameters (SB and $\mathrm{ZH}$ ) independently evaluated the vertebral body height in the anterior, middle and posterior portions as well as the local kyphotic angle (LKA) of the fractured vertebra on pre- and post-operative radiographs for all patients. Image analysis was carried out using Centricity Enterprise $\mathrm{Web}^{\circledR}{ }^{\circledR}$ (GE Healthcare, Buckinghamshire, UK). The mean values from both measurements were used for statistical analysis to minimize the risk of systematic bias. The height of the fractured vertebra was calculated as the relative height compared to the mean height of the nearest unfractured vertebrae proximally and distally to minimize the influence of varying magnifications. To assess the reposition that was achieved through kyphoplasty of the fractured vertebra, the difference between pre- and post-operative relative vertebral body height and between pre- and post-operative LKA were calculated. Example measurements are shown in Fig. 1.

\section{Clinical parameters}

Complementary to the radiological measurements, we retrieved clinical data on patient characteristics and the course of treatment from medical records. These data included age, sex, interval between injury and surgery, osteoporosis medication (calcium or Vitamin D supplements, 


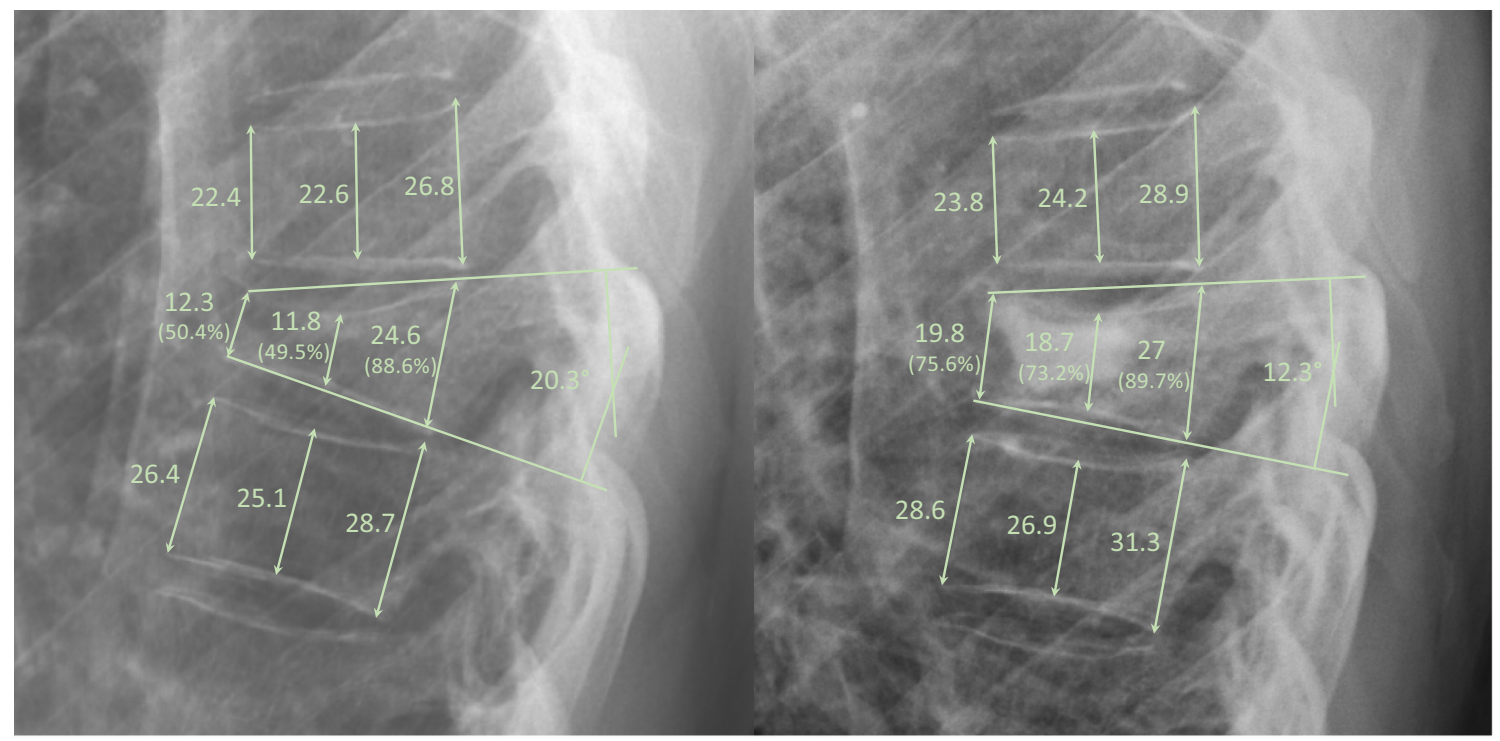

Fig. 1 Measurement of radiographic parameters on pre- (left) and post-operative (right) radiographs from a 47-year-old patient from the acute group. The restoration was $25.4 \%$ for the anterior portion,

bisphosphonates, teriparatide, Denosumab), pain on a visual analogue scale (VAS) at rest and in motion (1 day preoperatively and 2 days post-operatively), use of analgesics according to the WHO Analgesic Ladder (1, non-opioid analgesics; 2 , non-opioids + weak opioids; 3 , non-opioids + strong opioids; assessed 1 day preoperatively and 2 days post-operatively), body mass index (BMI) and post-operative day of full mobilization.

\section{Statistical analysis}

Patient characteristics are presented as mean values with standard deviation or percentages, as appropriate. Baseline characteristics between the three different groups were compared using a one-way analysis of variance (ANOVA). Differences between pre- and post-operative parameters within the groups were compared using a paired $t$-test for parametric variables and a Wilcoxon test for nonparametric variables, whereas an unpaired $t$-test (with Welch correction where necessary) for parametric variables was used to compare the means between two groups. Pearson's correlation coefficient was calculated to evaluate the correlation between operative timing and local height restoration.

\section{Results}

\section{Study population}

A total of 492 patients were treated using kyphoplasty at our institution between September 2012 and December
$23.7 \%$ for the middle portion, $1.1 \%$ for the posterior portion and $8^{\circ}$ for the local kyphotic angle

2018. Of these patients, 230 patients fulfilled the inclusion criteria (Fig. 2), and of these 230 patients, 100 had acute fractures, 91 had subacute fractures, and 39 had chronic fractures. Baseline characteristics of the study population are presented in Table 1. There were no significant

Patients treated
with Kyphoplasty
$(01 / 2012$ ó 12/2018)

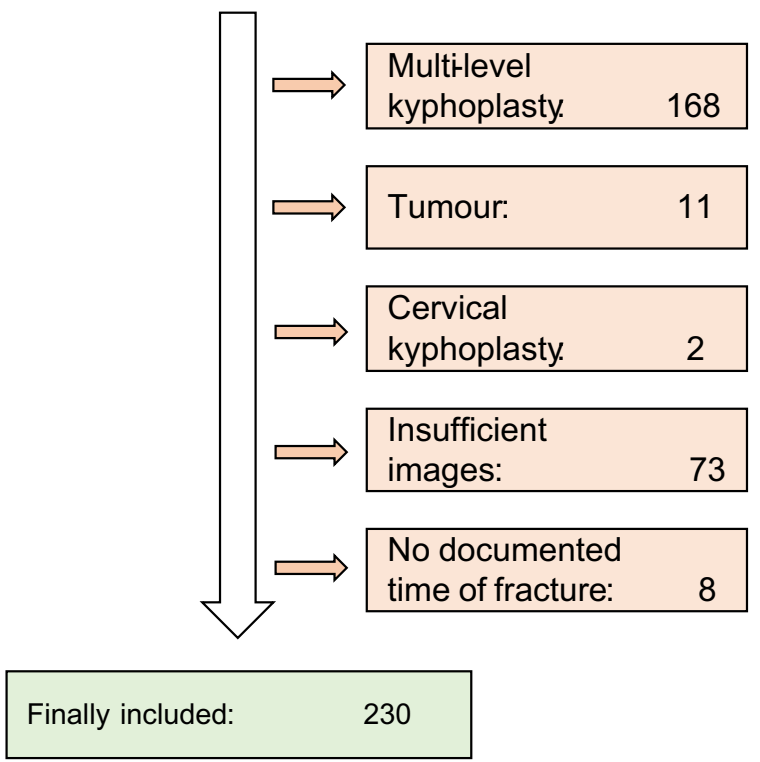

Fig. 2 Study flowchart 
Table 1 Characteristics of the study population

\begin{tabular}{lllllr}
\hline Parameter & All groups $( \pm$ SD) & Acute $( \pm$ SD) & Subacute $( \pm$ SD $)$ & Chronic $( \pm$ SD $)$ & $p$ \\
\hline$N$ & 230 & 100 & 91 & 39 & - \\
Sex & $69 \mathrm{~m} ; 161 \mathrm{w}$ & $27 \mathrm{~m} ; 73 \mathrm{w}$ & $30 \mathrm{~m} ; 61 \mathrm{w}$ & $12 \mathrm{~m} ; 27 \mathrm{w}$ & .666 \\
Age $($ years $)$ & $71.7( \pm 10.7)$ & $72.2( \pm 11.5)$ & $71.8( \pm 10.3)$ & $70.4( \pm 9.6)$ & .683 \\
Weight $(\mathrm{kg})$ & $71.3( \pm 15.1)$ & $70.2( \pm 15.9)$ & $72.2( \pm 15.7)$ & $72.2( \pm 11)$ & .633 \\
Height $(\mathrm{cm})$ & $167.3( \pm 8.8)$ & $166.7( \pm 8.8)$ & $168( \pm 8.8)$ & $167.6( \pm 8.4)$ & .530 \\
BMI $\left(\mathrm{kg} / \mathrm{m}^{2}\right)$ & $25.3( \pm 4.2)$ & $25.2( \pm 4.3)$ & $25.4( \pm 4.4)$ & $25.7( \pm 3.6)$ & .786 \\
VAS at rest $(n=221)$ & $3.9( \pm 2.4)$ & $4( \pm 2.1)$ & $3.9( \pm 2.6)$ & $3.4( \pm 2.3)$ & .350 \\
VAS in motion $(n=220)$ & $5.6( \pm 2.1)$ & $5.5( \pm 1.8)$ & $5.8( \pm 2.3)$ & $5.2( \pm 2.5)$ & .322 \\
Thoracic fractures & $92(40 \%)$ & $39(39 \%)$ & $40(44 \%)$ & $13(33.3 \%)$ & .511 \\
Lumbar fractures & $1318(60 \%)$ & $61(61 \%)$ & $51(56 \%)$ & $26(66.7 \%)$ & .511 \\
Cement volume $(\mathrm{ml})$ & $6.8( \pm 2)$ & $6.9( \pm 1.9)$ & $6.4( \pm 2.1)$ & $7.4( \pm 2)$ & .115 \\
Calcium & $31(13.5 \%)$ & $16(7 \%)$ & $13(5.7 \%)$ & $2(0.8 \%)$ & .234 \\
Vitamin D & $78(33.9 \%)$ & $30(13 \%)$ & $33(14.3 \%)$ & $15(6.5 \%)$ & .534 \\
Bisphosphonates & $32(13.9 \%)$ & $12(5.2 \%)$ & $12(5.2 \%)$ & $8(3.5 \%)$ & .417 \\
Denosumab & $2(0.9 \%)$ & $1(0.4 \%)$ & 0 & $1(0.4 \%)$ & .351 \\
Teriparatid & 0 & 0 & 0 & 0 & - \\
Time since Injury & $33.9( \pm 47.1)$ & $8.1( \pm 4.3)$ & $28.7( \pm 7.7)$ & $112.3( \pm 71.1)$ & - \\
\hline
\end{tabular}

differences regarding the baseline characteristics between the three study groups. Of these 230 patients, 92 presented with thoracic fractures (Th4: 1; Th5: 4; Th6: 5; Th7: 4; Th8: 11; Th9: 8; Th10: 4; Th11: 17; Th12: 38) and 138 with lumbar fractures (L1: 63; L2: 25; L3: 22; L4: 19; L5: $9)$. Patients received an average of $6.8 \mathrm{ml}( \pm 2)$ of bone cement during kyphoplasty and were discharged after a mean of $4.6( \pm 5.2)$ days post-operatively.

\section{Radiological outcome after kyphoplasty}

For the entire population as well as in each subgroup, there was a significant improvement in the anterior vertebral body height (AVBH), middle vertebral body height (MVBH) and LKA post-operatively (Table 2). On average, the LKA could be corrected by $3.7^{\circ}$, ranging from $1.7^{\circ}$ (chronic group) to $4.3^{\circ}$ (subacute group) depending on the age of the injury. The posterior vertebral body height restoration was lower than the changes of the other parameters in all groups, but still reached significance for the whole patient collective as well as the subacute subgroup. In addition, $52 / 100$ patients $(52 \%)$ from the acute group, $47 / 91$ patients (51.6\%) from the subacute group, and 14/39 patients $(35.9 \%)$ from the chronic group had a restoration of $\geq 10 \%$ in relation to the height of the adjacent vertebrae in the anterior portion. Inter-rater reliability was generally high (intra-class correlation coefficient for preoperative measurements: AVBH 0.97; MVBH 0.95; PVBH 0.89; LKA 0.96; for post-operative measurements: AVBH 0.93; MVBH 0.9; PVBH 0.78; LKA 0.95).
Table 2 Restoration of vertebral body height and local kyphotic angle

\begin{tabular}{|c|c|c|c|c|c|}
\hline \multirow[t]{2}{*}{ Parameter } & \multirow[t]{2}{*}{ Mean diff. [\%] } & \multirow[t]{2}{*}{$\begin{array}{l}\text { Standard } \\
\text { deviation }\end{array}$} & \multicolumn{2}{|c|}{$\begin{array}{l}95 \% \text { Confidence } \\
\text { interval }\end{array}$} & \multirow[t]{2}{*}{$p$} \\
\hline & & & Lower & Upper & \\
\hline \multicolumn{6}{|c|}{ All patients } \\
\hline AVBH & 11.3 & 13.8 & 9.5 & 13.1 & .000 \\
\hline MVBH & 11.9 & 13.9 & 10.1 & 13.7 & .000 \\
\hline PVBH & 1.8 & 8.4 & .7 & 2.9 & .001 \\
\hline LKA & 3.7 & 5.8 & 2.9 & 4.4 & .000 \\
\hline \multicolumn{6}{|l|}{ Acute } \\
\hline AVBH & 10.8 & 13.9 & 8 & 13.6 & .000 \\
\hline MVBH & 11.1 & 13.6 & 8 & 13.8 & .000 \\
\hline PVBH & 1.4 & 7.7 & -.1 & 2.9 & .076 \\
\hline LKA & 3.8 & 5.2 & 2.7 & 4.8 & .000 \\
\hline \multicolumn{6}{|l|}{ Subacute } \\
\hline AVBH & 12.9 & 14.7 & 9.9 & 16 & .000 \\
\hline MVBH & 13.4 & 13.1 & 10.7 & 16.1 & .000 \\
\hline PVBH & 2 & 8.1 & .3 & 3.6 & .024 \\
\hline LKA & 4.3 & 6.4 & 3 & 5.7 & .000 \\
\hline \multicolumn{6}{|l|}{ Chronic } \\
\hline AVBH & 8.9 & 10.9 & 5.3 & 12.4 & .000 \\
\hline MVBH & 10.7 & 16.4 & 5.4 & 16 & .000 \\
\hline PVBH & 2.5 & 10.8 & -1 & 6 & .160 \\
\hline LKA & 1.7 & 5.3 & .03 & 3.5 & .046 \\
\hline
\end{tabular}

$A V B H$ vertebral body height in the anterior portion; $M V B H$ vertebral body height in the middle portion; $P V B H$ vertebral body height in the posterior portion; $L K A$ local kyphotic angle 


\section{Clinical outcome after kyphoplasty}

Patients from all groups had significant pain reduction at rest (all patients: Z-score-10.1; acute: Z-score -7; subacute: Z-score: -6.3 ; chronic: Z-score -3.7 ; each $p<0.001)$ and in motion (all patients: Z-score-11.3; acute: Z-Score -7.8; subacute: Z-score-6.9; chronic: Z-score-4.8; each $p<0.001$ ) 2 days post-operatively (Fig. 3).

In patients with acute fractures, the use of analgesics according to the WHO pain ladder was significantly reduced 2 days post-operatively ( $\mathrm{Z}$-score $-2.2 ; p=0.027)$ and at the time of discharge $(\mathrm{Z}$-score $-3.3 ; p=0.001)$. In the subacute group, there was a trend towards less use of analgesics 2 days post-operatively, which was not significant (Z-score-1.6; $p=0.118$ ). At time of discharge, the use of analgesics was also significantly lower than preoperatively in the subacute group (Z-score $-3.2 ; p=0.001)$. For patients with chronic fractures, there was a trend towards a lower use of analgesics 2 days post-operatively ( $Z$-score $-1.4 ; p=0.177$ ) and at discharge (Z-score $-0.5 ; p=0.642)$, which was less pronounced than in the other two groups and did not reach significance.

Two hundred twenty-one out of 230 patients $(96.1 \%)$ were fully mobilized (or, in case of pre-existent disability, reached their preoperative level of mobility) within 1 day after surgery, and $100 \%$ of patients were fully mobilized within 5 days. Thirty-five patients $(15.2 \%)$ had cement leakage on post-operative radiographs, but none of the affected patients required any further intervention. Two patients $(0.9 \%)$ were monitored on the intensive care unit post-operatively (both due to respiratory insufficiency), but they recovered without any sequelae. None of the patients had spinal infections or fatal complications during the hospital stay.

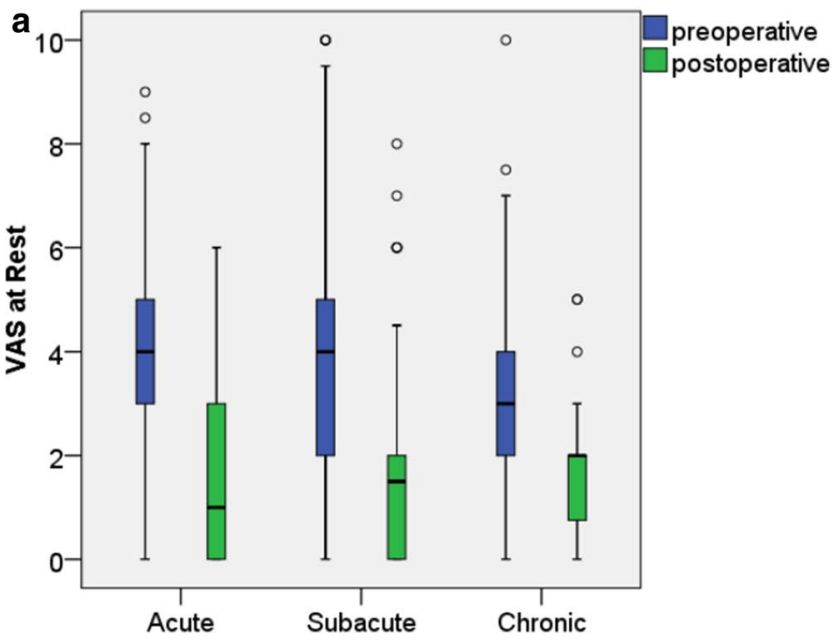

\section{Influence of operative timing on height restoration}

When comparing the reposition of the vertebral body between the different groups, patients with an interval of less than 6 weeks between injury and surgery (i.e. the acute and the subacute groups) showed a significantly higher increase in LKA post-operatively than patients from the chronic group (Table 3). Additionally, there was a trend towards for a higher restoration of $\mathrm{AVBH}$ and $\mathrm{MVBH}$ in

Table 3 Comparison of vertebral body height restoration between groups

\begin{tabular}{|c|c|c|c|c|}
\hline \multirow[t]{2}{*}{ Parameter } & \multirow[t]{2}{*}{ Mean diff } & \multicolumn{2}{|c|}{$95 \%$ Confidence interval } & \multirow[t]{2}{*}{$p$} \\
\hline & & Lower & Upper & \\
\hline \multicolumn{5}{|c|}{ Acute vs. subacute } \\
\hline AVBHR & -2.1 & -6.2 & 2 & .311 \\
\hline MVBHR & -2.4 & -6.2 & 1.5 & .225 \\
\hline PVBHR & -.58 & -2.8 & 1.7 & .618 \\
\hline LKAR & -.6 & -2.2 & 1.1 & .497 \\
\hline \multicolumn{5}{|c|}{ Acute vs. chronic } \\
\hline AVBHR & 2 & -2.5 & 6.4 & .385 \\
\hline MVBHR & .3 & -5 & 5.7 & .899 \\
\hline PVBHR & -1.1 & -4.3 & 2.2 & .510 \\
\hline LKAR & 2 & .1 & 4 & .042 \\
\hline \multicolumn{5}{|c|}{ Subacute vs. chronic } \\
\hline AVBHR & 4.1 & -.6 & 8.7 & .085 \\
\hline MVBHR & 2.7 & -2.7 & 8.1 & .320 \\
\hline PVBHR & -.5 & -3.9 & 2.9 & .767 \\
\hline LKAR & 2.6 & .3 & 4.9 & .027 \\
\hline
\end{tabular}

$A V B H R$ restoration of the vertebral body height in the anterior portion; $M V B H R$ restoration of the vertebral body height in the middle portion; $P V B H R$ restoration of the vertebral body height in the posterior portion; $L K A R$ restoration of the local kyphotic angle

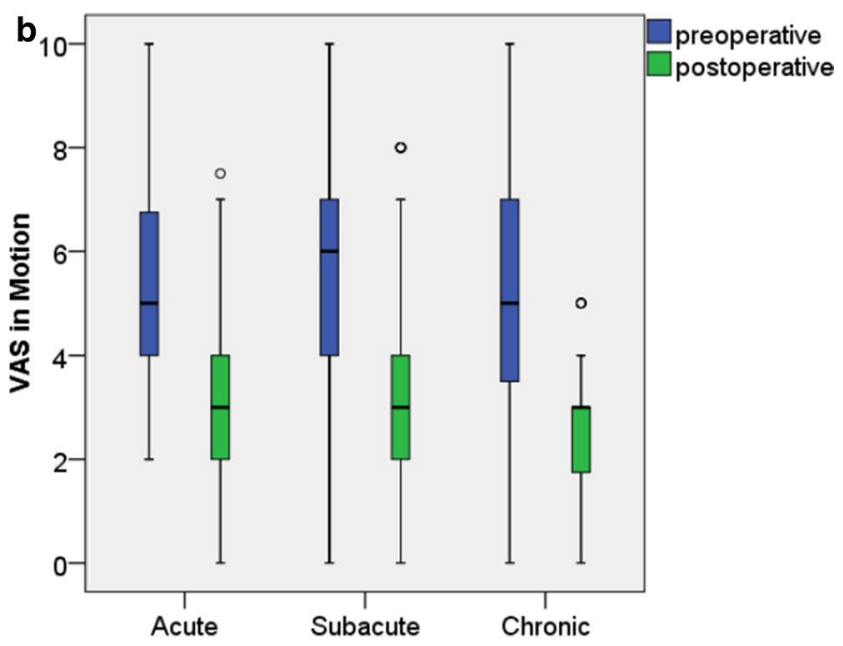

Fig. 3 Pre- and post-operative pain according to visual analogue scale (VAS) at rest (a) and in motion (b) one day before and two days after surgery 
the acute and subacute groups compared to the chronic group, which did not reach significance. There were no significant differences between the acute and the subacute groups. Regression analysis did not show a significant linear correlation between operative timing and restoration of LKA $(r=-0.66, p=0.318)$, AVBH $(r=-0.048, p=0.469)$, MVBH $(r=0.013, p=0.845)$ or PVBH $(r=-0.005$, $p=0.942)$.

\section{Discussion}

In the present study, we investigated the outcome after kyphoplasty in patients with VCFs and its dependence on the timing of the surgery. We could demonstrate that kyphoplasty results in a better local height restoration and a better reduction of analgesic consumption if performed within 6 weeks after fracture occurrence.

Previous studies have generally shown good pain relief and a low risk of complications for kyphoplasty $[15,16]$. This is supported by the findings of our study, where patients generally showed significant pain relief on the second postoperative day. Although cement leakage was radiologically observed in $14.3 \%$ of patients, none of the patients in our study showed any severe complications such as respiratory complications resulting from pulmonary embolism. The general notion that kyphoplasty is a rather minor and welltolerated intervention supported by the finding that more than $96 \%$ of patients were already fully mobilized within 1 day after surgery.

Additionally, our findings confirmed those of previous studies that reported good efficacy of kyphoplasty in restoring the vertebral body height after fractures [15, 17]. Vertebral body height in the anterior and middle portions improved significantly in all groups by around $11 \%$. Although 11\% might appear rather low at first sight, it should be noted that the height restoration refers to percentage of the expected pre-fracture height of the vertebral body. The percentage of reposition of the fractured vertebra itself is consequently higher than $11 \%$. In our study, the relative vertebral body height of the fractured vertebra was around $69 \%$ for both the anterior and middle portion preoperatively. Thus, a restoration of $11 \%$ of the expected normal height of the respective vertebral body equals a restoration of $35 \%$ of the fracture-related deformity. Of note, even in the chronic group, in which the fracture had occurred more than 110 days ago on average, there was still a significant height reposition in the anterior and middle portion.

To evaluate the influence of operative timing on the outcome after kyphoplasty, we divided the patients into three different groups according to the interval between symptom onset and surgical treatment. The baseline characteristics of the patients in the three groups were very similar and did not show any significant differences, which ensures good comparability.

Irrespective of the operative timing, patients from all groups benefitted from significant post-operative pain relief on the second post-operative day. For the use of analgesics, however, we observed differences between the groups. Patients from the acute group already took significantly fewer painkillers on the second post-operative day, whereas for patients with subacute fractures, the difference was only significant on the day of discharge, but not 2 days after surgery. Patients with chronic fractures also had a trend towards a lower use of analgesics post-operatively, but, on average, there was no significant reduction in the use of painkillers during the time of hospitalization, despite the significant reduction in pain according to the VAS scale. This observation might indicate that after taking painkillers for prolonged periods, patients can get used to them and struggle to stop their consumption. This is an important point to consider when deciding upon the optimal timing of surgery because any painkiller has potential side effects, such as permanent renal damage for NSAIDs or addiction for opioids. The results of our study suggest that for a surgery that occurs later, drug consumption is not only prolonged until the time of operation, but even further until after surgery. To the best of our knowledge, no other study has investigated the relationship between surgical timing and reduction of analgesic consumption.

The central question in our study was whether the timing of kyphoplasty influences the achievable restoration of the fracture-related loss in vertebral body height. This point is of high clinical relevance because deformity of the fractured vertebrae can cause various resulting issues. Due to the kyphotic deformity, the patient's centre of gravity is shifted anteriorly, leading to higher flexion bending moments around the apex of the kyphosis, which may induce pain and further increase the risk of additional fractures [18-20]. Additionally, VCF adverse effects on the quality of life, physical function, mental health and lifespan are related to the severity of the spinal deformity and are partly independent of pain [19-21]. Both the acute and the subacute groups showed significantly better restoration of the LKA than the chronic group. These findings are consistent with a previous study that compared the results in two groups of 20 patients each, which either received kyphoplasty within 2 weeks or after more than 2 weeks and reported a better restoration in the first group [20]. However, that study did not further investigate differences among the patients receiving kyphoplasty after $>2$ weeks. Two further studies including 51 and 36 patients came to the conclusion that early kyphoplasty within 4 weeks allows a better alignment than a later intervention [11, 12]. Neither of these studies investigated if there are further differences between an early and a very early intervention. 
Our results show that patients can still benefit from the full restoration potential even if the surgery is delayed for up to 6 weeks. This is of high clinical relevance because many patients primarily prefer a conservative treatment approach and 2 weeks might not be enough time for a final evaluation of this conservative treatment. Two additional retrospective studies on 72 and 99 patients who were treated with kyphoplasty also reported better restoration in patients who were treated within 2 months after injury. However, one study only reported absolute numbers for each group itself, but did not statistically examine if the differences between the groups were significant and therefore did not allow any reliable conclusions [22]. The other study only reported a better preservation of the anterior vertebral and kyphotic angle in the early group, but did not find significant absolute differences [23].

Despite our efforts to adhere to a rigorous methodology, there are some unavoidable limitations of our study. All radiographic measurements in our study where performed using plain radiographs, which represents the current clinical standard for routine pre- and post-operative imaging. However, the precision of measurements of radiographic parameters on plain radiographs is limited, resulting in a risk of measurement errors. CT images might theoretically offer a higher precision. Furthermore, many patients with osteoporotic fractures do not remember a specific trauma, which makes it challenging to determine the exact age of the fracture in some cases. Therefore, we have defined the time of injury as the time of symptom onset reported by the patient. Patients who did not report a specific time of symptom onset were excluded from the study. Still, it is possible that this approach did not result in a correct estimation of fracture age in all patients. Besides, this study is based on a retrospective data analysis with no prospective patient randomization. Although our baseline characteristics suggest good comparability of the three study groups, there might still be additional factors of bias. To the best of our knowledge, the 230 patients who were included in this study represent the largest population of such a study to date. However, the chronic fracture group was rather small with 39 patients. This is because of the regular treatment algorithms at our institutions, where we closely follow our patients during the first weeks and aim to make an early decision about conservative versus surgical treatment. Therefore, most patients that present soon after symptom onset will either undergo surgery within the first 6 weeks or will continue with conservative treatment. It might be conceivable that additional results could be obtained with larger cohorts. Additionally, we did not follow patients after discharge. Therefore, it is not possible to draw any reliable conclusions regarding the long-term effect of kyphoplasty, including the risk of a loss of correction or adjacent fractures. Prospective trials with a longer follow-up will be needed to determine the long-term effect of the height restoration achieved by kyphoplasty, which could not be shown by any study so far.

In conclusion, our results demonstrate that a better correction of fracture-related kyphotic deformation is possible if kyphoplasty is performed within 6 weeks after injury compared to surgery after $>6$ weeks. Therefore, we suggest aiming for a final decision upon conservative or surgical treatment during that period. However, if patients present with older fractures, kyphoplasty should still be considered as an option because significant pain reduction and vertebral height restoration are possible even after longer periods.

Acknowledgements Open Access funding provided by Projekt DEAL.

\section{Compliance with ethical standards}

Conflict of interest The authors declare that they have no conflict of interest.

Ethical approval The study has been approved by the institutional review board (EA1/203/18).

Open Access This article is licensed under a Creative Commons Attribution 4.0 International License, which permits use, sharing, adaptation, distribution and reproduction in any medium or format, as long as you give appropriate credit to the original author(s) and the source, provide a link to the Creative Commons licence, and indicate if changes were made. The images or other third party material in this article are included in the article's Creative Commons licence, unless indicated otherwise in a credit line to the material. If material is not included in the article's Creative Commons licence and your intended use is not permitted by statutory regulation or exceeds the permitted use, you will need to obtain permission directly from the copyright holder. To view a copy of this licence, visit http://creativecommons.org/licenses/by/4.0/.

\section{References}

1. Iqbal MM, Sobhan T (2002) Osteoporosis: a review. Mo Med 99:19-24

2. Johnell O (1996) Advances in osteoporosis: better identification of risk factors can reduce morbidity and mortality. J Intern Med 239:299-304. https://doi.org/10.1046/j.1365-2796.1996.42978 1000.x

3. Verbrugge LM, Lepkowski JM, Imanaka Y (1989) Comorbidity and its impact on disability. Milbank Q 67:450-484

4. Pisani P, Renna MD, Conversano F, Casciaro E, Di Paola M, Quarta E, Muratore M, Casciaro S (2016) Major osteoporotic fragility fractures: risk factor updates and societal impact. World J Orthop 7:171-181. https://doi.org/10.5312/wjo.v7.i3.171

5. Reginster JY, Burlet N (2006) Osteoporosis: a still increasing prevalence. Bone 38:S4-9. https://doi.org/10.1016/j.bone.2005.11.024

6. Johnell O, Kanis JA (2006) An estimate of the worldwide prevalence and disability associated with osteoporotic fractures. Osteoporos Int 17:1726-1733. https://doi.org/10.1007/s0019 8-006-0172-4

7. Wasnich RD (1996) Vertebral fracture epidemiology. Bone 18:179S-183S

8. Suzuki N, Ogikubo O, Hansson T (2009) The prognosis for pain, disability, activities of daily living and quality of life after an acute 
osteoporotic vertebral body fracture: its relation to fracture level, type of fracture and grade of fracture deformation. Eur Spine J 18:77-88. https://doi.org/10.1007/s00586-008-0847-y

9. Shah S, Goregaonkar AB (2016) Conservative management of osteoporotic vertebral fractures: a prospective study of thirty patients. Cureus 8:e542. https://doi.org/10.7759/cureus.542

10. Dachverband Osteologie (2017) Prophylaxe, diagnostik und therapie der osteoporose bei postmenopausalen frauen und bei männern. https://dv-osteologie.org/uploads/Leitlinie2017/FinaleVers ionLeitlinieOsteoporose2017_end.pdf . Last Accessed 10 May 2020

11. Minamide A, Maeda T, Yamada H, Murakami K, Okada M, Enyo Y, Nakagawa Y, Iwasaki H, Tsutsui S, Takami M, Nagata K, Hashizume H, Yukawa Y, Schoenfeld AJ, Simpson AK (2018) Early versus delayed kyphoplasty for thoracolumbar osteoporotic vertebral fractures: the effect of timing on clinical and radiographic outcomes and subsequent compression fractures. Clin Neurol Neurosurg 173:176-181. https://doi.org/10.1016/j.cline uro.2018.07.019

12. Zhou X, Meng X, Zhu H, Zhu Y, Yuan W (2019) Early versus late percutaneous kyphoplasty for treating osteoporotic vertebral compression fracture: a retrospective study. Clin Neurol Neurosurg 180:101-105. https://doi.org/10.1016/j.clineuro.2019.03.029

13. Cosman F, de Beur SJ, LeBoff MS, Lewiecki EM, Tanner B, Randall S, Lindsay R, National Osteoporosis F (2014) Clinician's guide to prevention and treatment of osteoporosis. Osteoporos Int 25:2359-2381. https://doi.org/10.1007/s00198-014-2794-2

14. Kanis JA, Cooper C, Rizzoli R, Reginster JY (2019) Scientific advisory board of the European society for C, Economic aspects of $\mathrm{O}$, the committees of Scientific A, national societies of the international osteoporosis F European guidance for the diagnosis and management of osteoporosis in postmenopausal women. Osteoporos Int 30:3-44. https://doi.org/10.1007/s0019 8-018-4704-5

15. Lieberman IH, Dudeney S, Reinhardt MK, Bell G (2001) Initial outcome and efficacy of "kyphoplasty" in the treatment of painful osteoporotic vertebral compression fractures. Spine (Phila Pa 1976) 26:1631-1638. https://doi.org/10.1097/00007632-20010 $7150-00026$
16. Garfin SR, Yuan HA, Reiley MA (2001) New technologies in spine: kyphoplasty and vertebroplasty for the treatment of painful osteoporotic compression fractures. Spine (Phila $\mathrm{Pa}$ 1976) 26:1511-1515. https://doi.org/10.1097/00007632-20010 7150-00002

17. Phillips FM, Ho E, Campbell-Hupp M, McNally T, Todd Wetzel F, Gupta P (2003) Early radiographic and clinical results of balloon kyphoplasty for the treatment of osteoporotic vertebral compression fractures. Spine 28:2260-2265. https://doi. org/10.1097/01.BRS.0000085092.84097.7B

18. Gold DT (1996) The clinical impact of vertebral fractures: quality of life in women with osteoporosis. Bone 18:185S-189S. https:// doi.org/10.1016/8756-3282(95)00500-5

19. Silverman SL (1992) The clinical consequences of vertebral compression fracture. Bone 13(Suppl 2):S27-31. https://doi. org/10.1016/8756-3282(92)90193-z

20. Park HTL, Lee CB, Ha JH, Choi SJ, Kim MS, Ha JM (2010) Results of kyphoplasty according to the operative timing. Current Orthopaedic Practice 21:489-493

21. Gold D, Lyles K (1999) Fractures: effects on quality of life. In: Bilezikian JP, Glowacki J, Rosen CJ (eds) The aging skeleton san diego. Academic Press, New York, pp 373-382

22. Oh GS, Kim HS, Ju CI, Kim SW, Lee SM, Shin H (2010) Comparison of the results of balloon kyphoplasty performed at different times after injury. J Korean Neurosurg Soc 47:199-202. https ://doi.org/10.3340/jkns.2010.47.3.199

23. Takahashi S, Hoshino M, Terai H, Toyoda H, Suzuki A, Tamai K, Watanabe K, Tsujio T, Yasuda H, Kono H, Sasaoka R, Dohzono S, Hayashi K, Ohyama S, Hori Y, Nakamura H (2018) Differences in short-term clinical and radiological outcomes depending on timing of balloon kyphoplasty for painful osteoporotic vertebral fracture. J Orthop Sci 23:51-56. https://doi.org/10.1016/j. jos.2017.09.019

Publisher's Note Springer Nature remains neutral with regard to jurisdictional claims in published maps and institutional affiliations. 